\title{
Effect of dietary sodium on the severity of bronchial asthma
}

\author{
D Lieberman, D Heimer
}

\begin{abstract}
Background A high sodium intake has been found to increase bronchial reactivity in men with asthma. The effects of change in sodium intake on peak flow rate have not been determined.

Methods The effect of changing dietary salt intake for two weeks on the severity of asthma as measured by peak expiratory flow (PEF) was studied in 17 patients with mild asthma in an open randomised crossover trial. PEF measurements were made by the patients in their own homes. Patients were placed on three levels of dietary sodium intake: normal, low, and high. Sodium intake was assessed by 24 hour urine collection.

Results The mean (SD) urine sodium was 147 (45), 84 (32), and 201 (73) $\mathrm{mmol}$ / 24 hours in the normal, low, and high sodium intake periods respectively. There were no significant differences in PEF or PEF amplitude (highest-lowest PEF), an index of asthma lability, between the three dietary salt periods.

Conclusion A low and high dietary salt intake for two weeks has no effect on peak expiratory flow in patients with mild asthma.
\end{abstract}

A low prevalence of asthma is found in some of the world's poorest areas, and low dietary salt intake has been implicated. ${ }^{1-3} \mathrm{~A}$ correlation between regional mortality from asthma and purchase of table salt per person has been reported in England and Wales. ${ }^{4}$ These two epidemiological observations triggered a search for the possible association between salt intake and bronchial reactivity. Burney et $a l^{5}$ reported that bronchial reactivity, as determined by a histamine challenge test, correlated strongly with 24 hour urinary sodium excretion in a community population after allowing for smoking and atopy. Javid $e t$ $a l^{6}$ found an increase in bronchial reactivity with increasing salt intake in asthmatic patients of both sexes but not in healthy subjects. In a recent controlled study, Burney et $a l^{7}$ found an increase in bronchial reactivity in association with a high salt intake in asthmatic men but not women. The correlation between bronchial reactivity measured by a bronchial challenge test and clinical severity of asthma is variable. ${ }^{8-10}$ We have tested the hypothesis that the amount of daily salt intake influences the severity and lability of clinical asthma as measured by peak expiratory flow (PEF) and the diurnal variation in PEF as measured by PEF amplitude (the difference between the highest and lowest value each day).

\section{Patients and methods}

Seventeen patients with mild asthma (nine men and eight women) who attend the pulmonary outpatient clinic at the Soroka Medical Center regularly participated in the study. Their mean age was 43 (range 27-62) years. All patients were normotensive and non-smokers, had a history of intermittent wheezing and a greater than $15 \%$ change in $\mathrm{FEV}_{1}$ either spontaneously or after inhalation of a $\beta_{2}$ agonist. All patients inhaled a $\beta_{2}$ agonist as needed. Five of the patients used inhaled corticosteroids (beclomethasone dipropionate $400 \mu \mathrm{g} /$ day). None had used oral corticosteroids in the three months before the study. Ten patients were taking a sustained release theophylline preparation $(300 \mathrm{mg}$ twice daily). The study was approved by the local human experimentation committee and written consent was obtained from all patients. To minimise bias, patients were told that the response of an individual to a change in dietary salt intake was unpredictable.

DIETS

Three regimens of dietary salt were tested, each for two weeks: normal diet-the patients were asked to continue their regular diet without any deliberate change in salt intake; low salt diet-the participants were put on a standard low salt diet used for hypertensive patients; high salt diet-the patients were asked to add as much salt as possible to their regular diet and to take four tablets of sodium chloride (500 mg) daily.

PEAK FLOW MEASUREMENT

Peak expiratory flow was measured at home with a mini peak flow meter (mini Wright). After instruction and training, patients were told to measure PEF three times daily throughout the study. The measurements were performed immediately after getting up in the morning, at noon, and before retiring at night, and the highest of three measurements was recorded. All measurements were performed before the use of a $\beta_{2}$ agonist. The mean values were calculated for each of the three daily measurements (morning, noon and night), for the whole day and for each 14 day period. The maximum difference between the

D Lieberman

Reprint requests to:

Dr Dov Heimer

Accepted 10 December 1991 
Table 1 Mean (SD) peak expiratory flow (PEF) in litres/min during the three dietary periods

\begin{tabular}{lcccccc}
\hline & \multicolumn{2}{c}{ Normal diet } & \multicolumn{2}{c}{ Low salt diet } & \multicolumn{2}{c}{ High salt diet } \\
\hline Morning & 370 & $(137)$ & 372 & $(139)$ & 368 & $(129)$ \\
Noon & 411 & $(137)$ & 410 & $(140)$ & 408 & $(138)$ \\
Night & 388 & $(136)$ & 399 & $(137)$ & 389 & $(134)$ \\
Daily mean & 389 & $(136)$ & 393 & $(138)$ & 388 & $(133)$ \\
\% of predicted & 71 & $(19)$ & 72 & $(19)$ & 71 & $(19)$ \\
PEF amplitude & 47 & $(24)$ & 44 & $(30)$ & 49 & $(30)$ \\
Inhaled $\beta_{2}$ agonist (puffs) & $3 \cdot 1(1 \cdot 9)$ & $3 \cdot 2(2)$ & $3 \cdot 6(2 \cdot 3)$ \\
\hline
\end{tabular}

All differences were non-significant.

Table 2 Confidence limits of differences

\begin{tabular}{llll}
\hline & Difference & $S D$ & $95 \%$ Confidence limits \\
\hline Peak flow & & & \\
ND $v$ LSD & 3.88 & $19 \cdot 05$ & $(-34 \cdot 22,+41.98)$ \\
LSD $v$ HSD & $-4 \cdot 18$ & $25 \cdot 72$ & $(-55 \cdot 62,+47 \cdot 26)$ \\
HSD $v$ ND & -0.29 & 21.63 & $(-43 \cdot 55,+42 \cdot 97)$ \\
& & & \\
$\beta_{2}$ Agonist usage & -0.15 & 1.04 & $(-2 \cdot 23,+1.93)$ \\
ND $v$ LSD & -0.33 & 1.39 & $(-3 \cdot 11,+2 \cdot 45)$ \\
LSD $v$ HSD & -0.5 & 2.05 & $(-4 \cdot 6,+3.6)$ \\
HSD $v$ ND & & & \\
\hline
\end{tabular}

ND—normal diet; LSD—low salt diet; HSD—high salt diet.

highest and lowest measurement of PEF each day (PEF amplitude) was used as an index of diurnal variation and asthma lability.

\section{URINE COLLECTION}

All participants were given verbal and written instruction on how to perform a 24 hour urine collection. The urine was collected on one of the last three days of each study period and brought to the chemical laboratory the following morning, where the volume was recorded and the sodium, potassium and creatinine concentration determined. Creatinine was measured by a modified Jaffe rate reaction. Sodium and potassium were measured by ion specific electrodes.

\section{STUDY PROTOCOL}

This was a randomised crossover open trial over six consecutive weeks. It was divided into three diet periods, each of two weeks, with no washout periods between the diet periods. Patients were assessed on entry into the study and at the end of each diet period. All participants started the study with the normal diet period, after which they were assigned at random to the low or high salt diet for the next two weeks. Thereafter they changed to the alternative diet for another two weeks. The remaining sodium chloride tablets were counted at the end of the high salt diet period as a measure of compliance. All participants had stable asthma during the month before the start of the study. The patients

Table 3 Mean (SD) concentrations of sodium, potassium and creatinine in 24 hour urine collections obtained in the three diet periods

\begin{tabular}{lrlrlrl}
\hline & \multicolumn{2}{c}{ Normal } & \multicolumn{2}{c}{ Low salt } & \multicolumn{2}{c}{ High salt } \\
\hline Sodium (mmol/24 hours) & 147 & $(45)$ & 84 & $(32)^{\star}$ & 201 & $(73)^{\star}$ \\
Potassium (mmol/24 hours) & 69 & $(20)$ & 75 & $(23)$ & 69 & $(20)$ \\
Creatinine (mg/24 hours) & 1371 & $(496)$ & 1411 & $(523)$ & 1384 & $(474)$ \\
\hline
\end{tabular}

${ }^{\star} \mathrm{p}<0.001$ were instructed not to change their usual medication during the study; $\beta_{2}$ agonist inhalers were used as needed and the number of puffs documented.

ANALYSIS

The measurements obtained from the three different diet periods were subjected to analysis of variance. Those which showed a significant difference were further tested by the paired $t$ test. A p value of $<0.05$ was regarded as significant. The $95 \%$ confidence intervals of the differences between mean values were calculated. The power of the study (17 subjects) to detect a $15 \%$ change in mean daily PEF was $24 \%$ based on a $5 \%$ statistical significance.

\section{Results}

No significant differences were found between the mean PEF values in the three periods (tables 1 and 2). No significant change was found when men and women were analysed separately. PEF amplitude did not differ between the three diet periods. The mean amplitude was about $12 \%$ of mean PEF (table 1).

The numbers of $\beta_{2}$ agonist inhalations were similar over the three diet periods (table 2 ).

Sodium excretion fell significantly from a mean (SD) of 147 (45) mmol/24 hours during the normal diet to 84 (32) $\mathrm{mmol} / 24$ hours on the low salt diet $(p<0.001)$ and increased significantly to 201 (73) $\mathrm{mmol} / 24$ hours on the high salt diet $(p<0.001)$ (table 3$)$. Potassium and creatinine excretion did not differ between the three diet periods. More than $85 \%$ of the tablets were used by the patients during the high salt diet period.

\section{Discussion}

In our study salt intake did not affect the severity of asthma in a small group of patients with stable asthma as measured by self monitoring of PEF. Our study was not blind as it is difficult to consume a high salt diet without the subject's awareness of the change. We tried to minimise directional expectations by explaining to the patients that the response to a low or high salt diet is individual and unpredictable. The measurements of sodium excretion indicate good compliance with the different diet protocols. The changes in salt excretion in our group are higher than those described by Burney et al. ${ }^{7}$ The trigger to our study was the observation that a high salt diet caused an increase in bronchial reactivity as measured by histamine challenge in men but not in women. ${ }^{67}$ In our study disease activity was measured on the basis of home PEF and not bronchial reactivity. Although there is some correlation between measures of reactivity and clinical disease severity, ${ }^{89}$ the two indices are not identical; a recent study reported poor correlation between these two indices. ${ }^{10}$ Diurnal variation of PEF can be used as an index of disease activity and bronchial lability. ${ }^{11}$ Self monitoring of disease 
activity with PEF has been shown to be a valuable and reliable tool. ${ }^{12}$ Our patients had mild asthma and were in a stable condition. This was confirmed by the relatively high PEF and the relatively low PEF amplitude, which represents the diurnal variation (about $12 \%$ of the mean).

Our study does not permit conclusions about the response of asthmatic patients with active disease or with more severe asthma. Possibly patients with more severe disease will have a greater sensitivity to the amount of salt intake. Increasing the power of the study by testing a larger group of patients might have detected small but significant changes in patients with mild asthma. However, the even distribution of the $95 \%$ confidence intervals of the differences in PEF and $\beta_{2}$ agonist usage around zero suggests that the likelihood of finding a difference between the three diets is very small.

1 Waite AD, Eyles EF, Tonkin SL, O'Donnell TV. Asthma prevalence in Tokelauan children in two environments. Clin Allergy 1980;10:71-5.

2 Van Niekerk CH, Weinberg EG, Shore SC, Heese HV, Van
Schalkwyk DJ. Prevalence of asthma: a comparative study of urban and rural Xhosa children. Clin Allergy 1979;9: 319-24.

3 Burney PGJ. The cause of asthma: does salt potentiate bronchial activity? JR Soc Med 1987;80:364-7.

4 Burney PGJ. A diet rich in sodium may potentiate asthma: epidemiological evidence for a new hypothesis. Chest
arney PGJ. A diet rich in sodium may potentiate asthma: 1987;91(suppl):143-8S.

5 Burney PGJ, Britton JR, Chinn S, Tattersfield AE, Platt HS, Papacosta AO, Kelson MC. Response to inhaled histamine and 24 hour sodium excretion. BMJ 1986;292: 1483-6.

6 Javid A, Cushley MJ, Bone MF. Effect of dietary salt on bronchial reactivity to histamine in asthma. $B M J 1988$; 297:454.

7 Burney PGJ, Neiled JE, Twort CH, Chinn S, Jones TD, Mitchell WD, et al. Effect of changing dietary sodium on airway response to histamine. Thorax $1989 ; 44: 36-41$.

8 Juniper EF, Frith PA, Hargreave FE. Airway responsiveness to histamine and methacholine: relationship to minimum treatment to control symptoms of asthma. Thorax 1981;36:575-9.

9 Ryan G, Latimer KM, Dolovich J, Hargreave FE. Bronchial responsiveness to histamine: relationship to diurnal variation of peak flow rate, improvement after bronchodilator, and airway caliber. Thorax 1982;37:423-9.

10 Pattemore PK, Innes Asher M, Harrison AC, Mitchell EA, Rea HH, Stewart AW. The interrelationship among bronchial hyperresponsiveness, the diagnosis of asthma, and asthma symptoms. Am Rev Respir Dis 1990;142: 549-54.

11 Hetzel MR, Clark TJH. Comparison of normal and asthmatic circardian rhythms in peak expiratory flow rate. Thorax 1980;35:732-8.

12 Hetzel MR, Williams IP, Shakespeare RM. Can patients keep their own peak-flow records reliably? Lancet 1979; i:597. 